\title{
Quench-produced solitons in a box-trapped Bose-Einstein condensate
}

\author{
E. J. Halperin $\odot$ and J. L. Bohn \\ JILA, NIST, and Department of Physics, University of Colorado, Boulder, Colorado 80309-0440, USA
}

(Received 24 February 2020; revised 29 April 2020; accepted 28 October 2020; published 19 November 2020)

\begin{abstract}
We describe a protocol to prepare solitons in a quasi-one-dimensional (quasi-1D) box-trapped Bose-Einstein condensate using a quench of the isotropic $s$-wave scattering length. A quench to exactly four times the initial 1D coupling strength creates one soliton at each boundary of the box, which then propagate in a uniform background density and collide with one another. In the ideal case, no nonsolitonic excitations are created during the quench. The procedure is robust against realistic box shapes, imperfections in the scattering length ramp rate, and a mismatch of the final scattering length. We simulate the condensate in full $3 \mathrm{D}$ and propose a modification to the protocol that is effective outside of the strict quasi-1D regime.
\end{abstract}

DOI: 10.1103/PhysRevResearch.2.043256

\section{INTRODUCTION}

Solitons appear in a variety of physical systems, such as shallow water, nonlinear optics, and interacting BoseEinstein condensates (BECs) [1]. Solitons, or "solitary waves," are localized modulations-whether of height, intensity, or density - which propagate without changing their shape or velocity. They are a hallmark of certain nonlinear systems. As opposed to phonons, which treat a nonlinear system as essentially linear, solitons rely on and demonstrate the nonlinear nature of the system. In a linear quantum system, such a density modulation would naturally disperse in a uniform background, and thus solitons in a BEC represent a compromise between interatomic interactions and generic wave-packet dispersion. Solitons provide an avenue for exploring manifestly nonlinear properties of interacting Bose gases.

As a result, a great deal of effort has been put into producing solitons in a laboratory setting. Several ingenious methods have been devised and experimentally implemented for making solitons in a quasi-one-dimensional (quasi-1D) Bose gas. The prevailing method for creating dark solitons is phase imprinting in a harmonically trapped BEC via carefully controlled laser fields $[2,3]$. Here the condensate is phase shifted by pulsing on the potential to create a phase jump. The imprinted phase discontinuity generates solitons as well as other structures [2] in the gas, as the bulk of the gas shifts in response to the half-raised potential, causing a sloshing mode. This method is somewhat limited by the resolution of the laser light used to imprint the phase and by the influence of this laser on the condensate density.

Published by the American Physical Society under the terms of the Creative Commons Attribution 4.0 International license. Further distribution of this work must maintain attribution to the author(s) and the published article's title, journal citation, and DOI.
Solitons have also been formed by directly controlling the condensate density via creating shock waves, which then create solitons and other excitations as they evolve [4], as well as by colliding two initially separated BECs [5]. Additionally, bright solitons, which consist of regions of higher than background density, have also been formed via quenches to negative scattering lengths $[6,7]$.

In general, these methods create excitations that are characteristic of, although often not exactly, solitons, and in turn lead to additional excitations. This effect has been mitigated by a recent experimental innovation that imprints both phase and density in the condensate [8]. This method produces relatively clean solitons with controllable velocities and a small degree of attendant nonsolitonic excitations.

In this article, we take advantage of two significant experimental developments-box traps $[9,10]$ and the ability to suddenly change the scattering length [11] - to propose a clean and experimentally convenient protocol for preparing solitons. Unlike phase-imprinting methods, our scheme does not require the careful engineering of the correct solitonic phase and density, but rather exploits the black-soliton-like profile intrinsic at the edge of a BEC confined to a box trap $[9,10]$. This profile has built into it the needed relation between phase and density profiles and is robust against imperfections in the box potential. Under a suitable quench of the scattering length, this feature creates solitons moving with a specified velocity away from the edges of the trap.

The method originates in a fascinating mathematical prediction from inverse scattering theory $[12,13]$, which posits that in a quench of the coupling strength $g$ from $g \rightarrow n^{2} g$, where $n$ is an integer, a soliton in an infinite uniform density BEC splits into $2 n-1$ solitons. This theory predicts that in a quench to any value other than a perfect square multiple of the initial scattering length, the quench will create solitons along with additional excitations. An interesting special case of this prediction concerns a black soliton, which is stationary and causes the density to go to zero at its center. After a quench in a uniform gas of a black soliton, the inverse scattering theory predicts that there will be $n-1$ solitons moving to the left 
and $n-1$ to the right, with one, now narrower, black soliton remaining stationary in the original location.

Motivated by this mathematical result, we propose an operationally straightforward and robust way to create solitons in a box-trapped BEC. The ground-state density of a large box-trapped BEC must go from nearly uniform in the center to zero at the edges, and does so such that the wave function looks exactly like half of a black soliton. Upon a quench of the ground-state BEC in a box from $g \rightarrow 4 g$, each "half soliton" at the boundary of the box generates one gray soliton, which is launched towards the center of the trap. No other excitations are created during the idealized quench; aside from the two traveling solitons and the boundary conditions, the gas presents a uniform background density. After verifying the efficacy of the procedure numerically, we discuss the robustness to experimental considerations. In particular, we look at realistic box shapes, finite ramp times, and quenches to a value that is close to, but not exactly, $4 g$. We then turn to a full 3D discussion of the protocol and present a method to offset transverse breathing modes caused during the quench via a simultaneous quench of the strength of the transverse trapping potential [14].

\section{DEMONSTRATION OF THE PROTOCOL}

We first numerically verify the inverse scattering theory result $[12,13]$ for a soliton in a uniform BEC. We simulate the Gross-Pitaevskii equation in a box with either periodic or hard-wall boundary conditions, which in 1D is given by

$$
i \hbar \frac{\partial \Psi}{\partial t}=\left[-\frac{\hbar^{2}}{2 m} \frac{\partial^{2}}{\partial x^{2}}+V+g|\Psi|^{2}\right] \Psi,
$$

where $\Psi$ is the many-body order parameter of the $N$-particle Bose gas, related to the single-particle wave function $\phi$ by $\Psi=\sqrt{N} \phi$. In 1D, $\Psi$ describes the linear number density. We set the external potential $V(x)=0$ and $g$ the $1 \mathrm{D}$ coupling constant, which is related to the 3D scattering length $a$ by $[15,16]$

$$
g=\frac{2 \hbar^{2} a}{m a_{\rho}^{2}} \frac{1}{1-A a_{\rho} / a},
$$

with $A \approx 1.03$, and $a_{\rho}$ the oscillator length in the transverse direction. If $0<a \ll a_{\rho}$, then $g \propto a$, so in this limit a quench of the $3 \mathrm{D}$ scattering length by a factor of 4 corresponds to a quench of the 1D coupling strength by a factor of 4 . In general, for a quench to four times the initial coupling strength, the required final 3D scattering length $a_{f}$ in terms of the initial $3 \mathrm{D}$ scattering length $a$ is

$$
a_{f}=\frac{4 a_{\rho} a}{a_{\rho}+3 A a},
$$

which reduces to $a_{f}=4 a$ in the case of $a \ll a_{\rho}$.

We find the ground-state solution of Eq. (1) via imaginarytime evolution [17] and evolve the solution in time using a time-splitting pseudospectral method [18]. The wave function for a single soliton in a uniform BEC has the form [19]

$$
\psi=\sqrt{n}\left[i \frac{v}{c}+\sqrt{1-\frac{v^{2}}{c^{2}}} \tanh \left(\frac{x-v t}{\sqrt{2} \xi} \sqrt{1-\frac{v^{2}}{c^{2}}}\right)\right],
$$
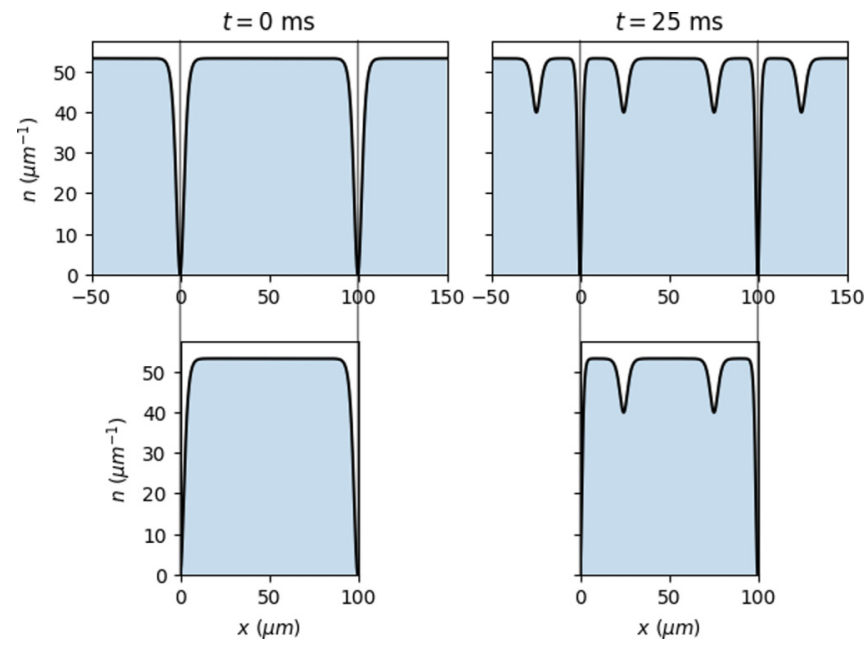

FIG. 1. Comparison of the dynamics of two black solitons after a quench in a uniform BEC (top) to the ground state in a box trap following the same quench (bottom), shown before (left) and $25 \mathrm{~ms}$ after (right) a quench to four times the initial coupling strength, with parameters as described in the text. The density in the box trap exactly matches that in the uniform gas, both before and after the quench within the boundaries of the box. Gray lines emphasize this correspondence.

with $n$ the background density, $v$ the velocity, $c=\sqrt{g n / m}$ the speed of sound, and $\xi=\hbar /(\sqrt{m g n})$ the healing length (which is sometimes defined with a factor of $\sqrt{2}$ in the denominator). The soliton is entirely determined by the single parameter $v$, which fixes both its velocity and depth. It is well known that solitons are unstable outside of the quasi-1D regime, where they decay into vortices and other excitations. We thus need to be in the regime where $N a / L<0.6[1,20]$ and $a_{\rho} / L \ll 1$, in which the soliton will be stable and the gas will be quasi1D. The finite width does, however, have implications for the scattering length quench, which we will address below. Although the idealized protocol is independent of the specific parameters, here we solve the Gross-Pitaevskii equation in a $100 \mu \mathrm{m}$ box, and a healing length of $\xi=3.1 \mu \mathrm{m}$. This can be achieved, for example, with $5000{ }^{39} \mathrm{~K}$ atoms at $a_{3 d}=20 a_{0}$ with $a_{\rho}=1 \mu \mathrm{m}$. Here, $N a / L=0.05$ and $a_{\rho} / L=0.005$. The speed of sound $c=0.5 \mathrm{~mm} / \mathrm{s}$, and the final $3 \mathrm{D}$ scattering length that gives four times the initial 1D coupling strength is $a_{f}=79.7 a_{0}$, as found via Eq. (3).

Figure 1 (top left) shows two black solitons in a uniform BEC, well separated by a distance $L=100 \mu \mathrm{m}$, with one at $x=0$ and one at $x=L$. The solitons are far enough apart that they are essentially independent and are well described by Eq. (4). Each soliton splits into three solitons following a quench from $g \rightarrow 4 g$. This is shown at a later time (top right). Inverse scattering theory gives quantitative predictions for the shape and velocity, with which our numerics agree to one part in $10^{4}$. The newly created solitons have a minimum density that is $3 / 4$ of the background density and move at $\sqrt{3} / 2$ of the final speed of sound, as theoretically predicted [12]. It is important to make a distinction here between the effective background density and the average density, which are not the same in a box. The solitons propagate in a background density 
that is slightly higher than the average density due to the finite nature of the box. This can be seen in Fig. 1, as the average density is $50 \mu \mathrm{m}^{-1}$, but the background density is seen to be $53 \mu \mathrm{m}^{-1}$.

This quench protocol maps directly onto a finite box trap. In the bottom row of Fig. 1, we show simulated density profiles for a box-trapped BEC, before (bottom left) and $25 \mathrm{~ms}$ after (bottom right) a quench from $g \rightarrow 4 g$. One can see how the density profile of the two solitons in the uniform gas exactly matches that of the box-trapped BEC, both before and after the quench. Furthermore, the narrower black solitons, without the newly created gray solitons, then match the ground state of the more strongly interacting gas near the boundaries of the box. From Fig. 1, the correspondence between the two scenarios is evident. The dynamics of the solitons in the uniform density BEC preserves the boundary conditions of the box at all times. Thus, using a "method of images," we can exactly map the problem of two black solitons separated by distance $L$ in a uniform BEC to the ground state of a trapped BEC in a box of length $L$. In this way, one may produce solitons in a box by an isotropic quench of the 1D coupling strength.

\section{ROBUSTNESS TO EXPERIMENTAL CONSIDERATIONS}

One obvious advantage of the method proposed here is that the phase and density profiles of the initial black solitons are automatically determined from the ground state of the initial condensate, without the need for careful engineering. These profiles are perfect in the limit of a pure box potential, but with some deviation given that a true experimental box must have walls that are Gaussians with width $\delta$. In this section, we therefore investigate the errors produced in this imperfect box, as well as errors due to finite quench times and mismatch in the final coupling strength.

There are two main types of error produced when the conditions of the protocol are not perfectly met. First, the solitons may not be the correct width or depth; second, there are density modulations in the bulk due to the production of phonon modes. We find the former to be a more sensitive measure of error, and fit each imperfect gray soliton to a function of the form

$$
\psi(x ; d, w)=\sqrt{n}\left[i \sqrt{1-d}+\sqrt{d} \tanh \left(\sqrt{\frac{3}{8}} \frac{x-v t}{\xi_{f} w}\right)\right],
$$

with $\xi_{f}$ the final healing length. As opposed to Eq. (4), the term inside the hyperbolic tangent is independent of $d$ in order to decouple variations in width and depth. The ideal soliton has $w=1$ and $d=0.25$. We quantify the error in width $\Delta w$ and height $\Delta d$ simply as the fractional error from the predicted values, i.e., $\Delta w=w-1$ and $\Delta d=4(d-0.25)$. When $\Delta d$ is positive (negative), the soliton is deeper (shallower) than expected, and when $\Delta w$ is positive (negative), the soliton is wider (narrower) than expected.

To track what is likely the main source of imperfection, namely, the influence of a rounded box on the initial black soliton, we simulate a box with Gaussian potential walls as

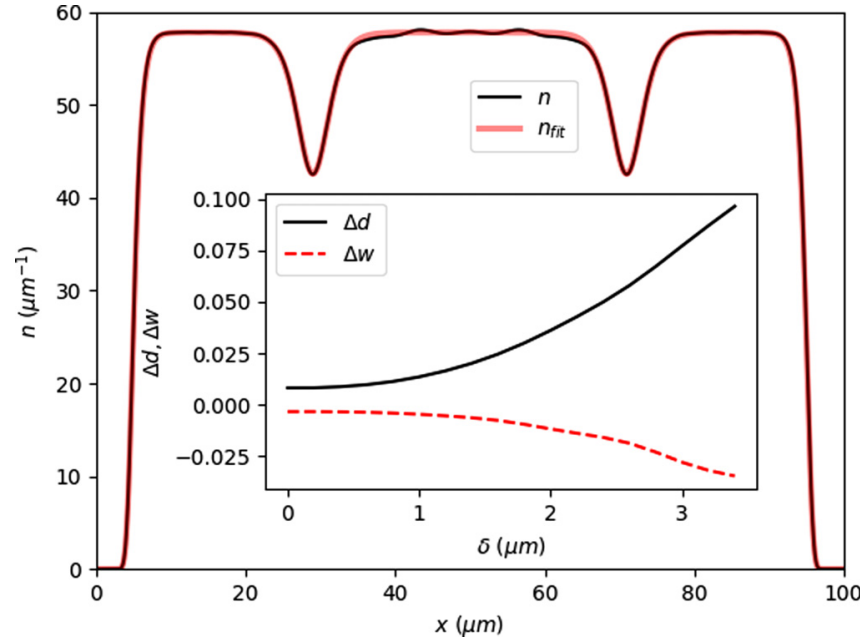

FIG. 2. The density profile $25 \mathrm{~ms}$ after quench from $g \rightarrow 4.04 g$ in a $100 \mu \mathrm{m}$ box with 2.5 - $\mu \mathrm{m}$-thick walls, and a quench time of $100 \mu \mathrm{s}$. The inset shows the variations in the expected soliton height and width as a function of the $1 / e^{2}$ width of the walls, using a $100 \mu \mathrm{s}$ quench time and $1 \%$ scattering length mismatch.

in $[9,10]$, and vary the $1 / e^{2}$ width $\delta$ of the Gaussians to investigate this effect. In addition, the generation of gray solitons may suffer imperfections from the quench not occurring instantaneously, or quenching to not quite the right final scattering length. In this context, note that recent quench experiments $[11,21]$ perform quenches as fast as $2-10 \mu \mathrm{s}$, with less than $1 \%$ mismatch in the final magnetic field. Therefore, in the simulations below, we will incorporate a final coupling that is $1 \%$ larger than the ideal value, and a quench duration of $100 \mu \mathrm{s}$.

Figure 2 shows an example of the density profile following a finite and imperfect quench (solid black line), in a box with $\delta=2.5 \mu \mathrm{m}$, along with the best-fit function that we use to calculate $\Delta w$ and $\Delta d$ (shaded red line). We evaluate the fractional error in width and depth after $25 \mathrm{~ms}$, when the solitons are well separated from each other and the boundaries. For the density profile shown in Fig. 2, we find $\Delta w=-0.02$ and $\Delta d=0.05$. Thus the excitations are well described by something solitonic; however, the solitons are slightly deeper and narrower than predicted. One can also see slight density modulations in the middle of the gas, showing phonon modes created during the realistic 1D quench. The maximum deviation of these phonon excitations from the background density amounts to $0.5 \%$.

The inset of Fig. 2 shows the deviations from the predicted width (dashed red line) and depth (solid black line) of the solitons as a function of the $1 / e^{2}$ width of the walls, using a constant height of $2 \mu \mathrm{K}$. For a perfect box, there are still slight errors caused by the finite quench time and scattering length mismatch. These small errors are exacerbated by the box shape; however, they remain quite small for realistic box sizes.

\section{3D CONSIDERATIONS}

This discussion so far demonstrates the efficacy of the protocol within the quasi-1D regime. For a sufficiently tightly 


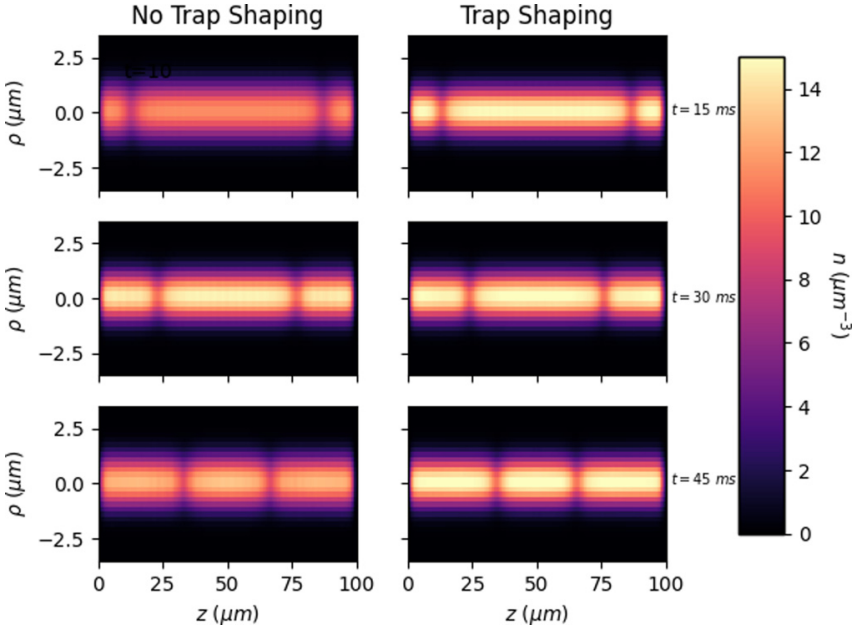

FIG. 3. Full 3D simulation of the protocol, showing a slice of the 3D density. On the left, we quench as in the 1D section, whereas on the right, we additionally quench the transverse trapping potential to offset the breathing mode. This density slice is shown for three different times in each case, which is different from the transverse breathing frequency of $0.6 \mathrm{~ms}$. The trap shaping nearly eliminates the transverse dynamics.

confined trap, the propagation of the solitons is expected to be essentially one dimensional. However, the act of quenching the scattering length will necessarily instigate breathing oscillations in the transverse direction, unless $N a_{f} / L \ll 1$. This breathing mode can be seen on the left in Fig. 6, where the density in the center of the trap is changing with time. In order to suppress this effect, we can simultaneously quench the trapping strength, proportional to the laser intensity, in the transverse direction, so that the prequench ground state in the original trap matches the postquench ground state in the final trap. This is a method that has been proposed to minimize the impact of a scattering length quench on the collective dynamics of the BEC [14]. Within a Gaussian ansatz for the ground state, we find that the final trap frequency $\omega_{f}$ is

$$
\omega_{f}=\sqrt{\frac{1+2 N a_{f} / L}{1+2 N a_{i} / L}} \omega_{i},
$$

where $a_{i}$ and $a_{f}$ are the initial and final 3D scattering lengths, respectively. $N$ is the total atom number, $L$ is the box length, and $\omega_{i}$ is the initial trap frequency. For our parameters, $\omega_{f} \approx$ $1.13 \omega_{i}$.

We show the protocol with and without a quench of the trapping potential in Fig. 3. Three different times slices are shown, for $15 \mathrm{~ms}$ (top), $30 \mathrm{~ms}$ (middle), and $45 \mathrm{~ms}$ (bottom), for a quench of only the 3D scattering length (left) and for a quench of both the scattering length and transverse trapping strength according to Eq. (3). In both cases, the solitons progress through the bulk of the gas, although on the left there is a transverse breathing mode created during the quench. This breathing mode leads to errors in the solitons that are produced as the solitons travel through a time-varying density, causing them to expand and contract as they move axially. This breathing mode is nearly absent in the shaped trap on the right.

Having discussed errors caused by the 3D case in isolation, we additionally test a superposition of all the errors discussed in the previous section with a 3D gas. We use an imperfect 3D box with $\delta=2.5 \mu \mathrm{m}$, a $100 \mu$ s quench time, and $1 \%$ coupling strength error. We find that for the integrated density profile, $\Delta d=0.02$ and $\Delta w=-0.07$ after $25 \mathrm{~ms}$. Coupling into the transverse direction tends to make the solitons shallower, whereas the imperfect box makes them deeper. These errors offset somewhat here. The solitons are minimally affected, even when several sources of error are present.

\section{CONCLUSION}

We have proposed and numerically verified a protocol to create solitons in a box using a quench of the coupling strength to four times its initial value. This protocol is seen to be robust to three possible sources of error in 1D. The protocol works well in 3D, where a simultaneous quench of the transverse trapping strength significantly reduces any errors here. Here there is no need to dynamically imprint any specific phase and density; the seed for the solitons is simply present in the ground state of the system. One is essentially only limited by the box shape, which is one controllable, time-independent field. The time-dependent part of the protocol, the B-field quench, is entirely isotropic. Errors induced by the quench itself are shown to be quite small. Although the protocol as discussed above produces a soliton with one specific shape, one could use a slight modification of the protocol to create soliton trains by quenching to another perfect square of the initial coupling strength. For example, a quench from $g \rightarrow 9 g$ would create two different gray solitons at each boundary, and $g \rightarrow 16 g$ would create three. In this way, one could systematically study several different solitons in the same parameter regime.

\section{ACKNOWLEDGMENTS}

This material is based upon work supported by the National Science Foundation under Grants No. PHY 1734006 and No. PHY 1806971. We gratefully acknowledge the suggestion of the anonymous referee to investigate the breakdown of the quasi-1D approximation.
[1] D. J. Frantzeskakis, Dark solitons in atomic Bose-Einstein condensates: From theory to experiments, J. Phys. A: Math. Theor. 43, 213001 (2010).

[2] S. Burger, K. Bongs, S. Dettmer, W. Ertmer, K. Sengstock, A. Sanpera, G. V. Shlyapnikov, and M. Lewenstein, Dark
Solitons in Bose-Einstein Condensates, Phys. Rev. Lett. 83, 5198 (1999).

[3] J. Denschlag, J. E. Simsarian, D. L. Feder, C. W. Clark, L. A. Collins, J. Cubizolles, L. Deng, E. W. Hagley, K. Helmerson, W. P. Reinhardt et al., Generating solitons by phase 
engineering of a Bose-Einstein condensate, Science 287, 97 (2000).

[4] Z. Dutton, M. Budde, C. Slowe, and L. V. Hau, Observation of quantum shock waves created with ultra-compressed slow light pulses in a Bose-Einstein condensate, Science 293, 663 (2001).

[5] A. Weller, J. P. Ronzheimer, C. Gross, J. Esteve, M. K. Oberthaler, D. J. Frantzeskakis, G. Theocharis, and P. G. Kevrekidis, Experimental Observation of Oscillating and Interacting Matter Wave Dark Solitons, Phys. Rev. Lett. 101, 130401 (2008).

[6] K. E. Strecker, G. B. Partridge, A. G. Truscott, and R. G. Hulet, Bright matter wave solitons in Bose-Einstein condensates, New J. Phys. 5, 73 (2003).

[7] L. D. Carr and J. Brand, Pulsed atomic soliton laser, Phys. Rev. A 70, 033607 (2004).

[8] A. R. Fritsch, M. Lu, G.H. Reid, A. M. Piñeiro, and I. B. Spielman, Creating solitons with controllable and near-zero velocity in Bose-Einstein condensates, Phys. Rev. A 101, 053629 (2020).

[9] A. L. Gaunt, T. F. Schmidutz, I. Gotlibovych, R. P. Smith, and Z. Hadzibabic, Bose-Einstein condensation of atoms in a uniform potential, Phy. Rev. Lett. 110, 200406 (2013).

[10] T. P. Meyrath, F. Schreck, J. L. Hanssen, C.-S. Chuu, and M. G. Raizen, Bose-Einstein condensate in a box, Phys. Rev. A 71, 041604(R) (2005).

[11] P. Makotyn, C. E. Klauss, D. L. Goldberger, E. A. Cornell, and D. S. Jin, Universal dynamics of a degenerate unitary Bose gas, Nat. Phys. 10, 116 (2014).
[12] O. Gamayun, Y. V. Bezvershenko, and V. Cheianov, Fate of a gray soliton in a quenched Bose-Einstein condensate, Phys. Rev. A 91, 031605(R) (2015).

[13] F. Franchini, A. Gromov, M. Kulkarni, and A. Trombettoni, Universal dynamics of a soliton after an interaction quench, J. Phys. A: Math. Theor. 48, 28FT01 (2015).

[14] E. J. Halperin, M. W. C. Sze, J. P. Corson, and J. L. Bohn, Stable production of a strongly interacting Bose-Einstein condensate via mode matching, Phys. Rev. A 100, 013608 (2019).

[15] M. Olshanii, Atomic Scattering in the Presence of an External Confinement and a Gas of Impenetrable Bosons, Phys. Rev. Lett. 81, 938 (1998).

[16] G. E. Astrakharchik, D. Blume, S. Giorgini, and B. E. Granger, Quasi-One-Dimensional Bose Gases with a Large Scattering Length, Phys. Rev. Lett. 92, 030402 (2004).

[17] M. L. Chiofalo, S. Succi, and M. P. Tosi, Ground state of trapped interacting Bose-Einstein condensates by an explicit imaginarytime algorithm, Phys. Rev. E 62, 7438 (2000).

[18] W. Bao and Y. Cai, Mathematical theory and numerical methods for Bose-Einstein condensation, Kinetic Relat. Mod. 6, 1 (2013).

[19] L. Pitaevskii and S. Stringari, Bose-Einstein Condensation (Oxford Science, New York, 2003).

[20] A. E. Muryshev, H. B. van Linden van den Heuvell, and G. V. Shlyapnikov, Stability of standing matter waves in a trap, Phys. Rev. A 60, R2665 (1999).

[21] C. Eigen, J. A. P. Glidden, R. Lopes, E. A. Cornell, R. P. Smith, and Z. Hadzibabic, Universal prethermal dynamics of Bose gases quenched to unitarity, Nature (London) 563, 221 (2018). 